\title{
The Quantum Arnold Transformation and the Ermakov-Pinney equation
}

\author{
Julio Guerrero ${ }^{1}$, Francisco F. López-Ruiz ${ }^{2}$ \\ ${ }^{1}$ Departamento de Matemática Aplicada, Universidad de Murcia, \\ Campus de Espinardo, 30100 Murcia, Spain. \\ 2 Departamento de Física Aplicada, Universidad de Cádiz, \\ Campus de Puerto Real, 11510 Puerto Real, Cádiz, Spain. \\ juguerre@um.es paco.lopezruiz@iaa.es
}

\begin{abstract}
The previously introduced Quantum Arnold Transformation, a unitary operator mapping the solutions of the Schrödinger equation for time dependent quadratic Hamiltonians into the solutions for the free particle, is revised and some interesting extensions are introduced, providing in particular a generalization of the Ermakov-Pinney equation.
\end{abstract}

\section{Introduction}

In [1], V.I. Arnold introduced a theorem stating that the family of graphs of solutions of any linear second-order differential equation (LSODE), with arbitrary time dependent coefficients, is locally diffeomorphic to the family of graphs of solutions of the simplest one-dimensional equation of motion, i.e., the equation of motion of a free particle. This important result allows to establish a direct relationship between the symmetries of an arbitrary classical linear system, whose equation of motion is a LSODE, with that of the free particle, which is known to have the largest possible symmetry group, $S L(3, \mathbb{R})$. The diffeomorphism was explicitly given in [1], namely the Classical Arnold Transformation (CAT), and can be seen as a particular class of more general Lie transformations relating systems defined by second order differential equations with the free particle [2].

In [3], the authors, in collaboration with V. Aldaya and F. Cossío, introduced the quantum version of the CAT, the Quantum Arnold Transformation (henceforth QAT), as a unitary map that relates the Hilbert space of solutions of the time-dependent Schrödinger equation for a Generalized Caldirola-Kanai oscillator (the quantum version of a classical system whose equation of motion is a LSODE) into the corresponding one for the free particle. In the present paper we revise the QAT and extend it in two respects. First, we give the explicit transformation connecting quantum physical systems with arbitrary time-dependent quadratic Hamiltonians with the free particle system, here called the Gauged QAT. Second and more importantly, we describe the transformation that links any LSODE-system to any other LSODE-system in terms of CAT's, giving rise to a generalization of the Ermakov-Pinney equation, and for that reason we shall call it the Arnold-Ermakov-Pinney transformation, providing also its quantum version.

Although the QAT is very recent, the CAT is more than thirty years old and, since it was introduced, many authors have used it (even without realizing it) and have built something similar to the QAT. Even before that, Lewis and Riesenfeld (1969) [4] introduced a technique to obtain solutions of the time-dependent Schrödinger equation (TDSE) for a time-dependent 
quadratic Hamiltonian (TDQH) as eigenfunctions of quadratic invariants. For that purpose they wrote the solutions in terms of auxiliary variables that satisfy the classical equations of motion (something that resembles the CAT). Dodonov \& Man'ko (1979) [5] constructed invariant operators for the damped harmonic oscillator and introduced coherent states, using a method similar to that of Lewis and Riesenfeld. Jackiw (1980) [6] gave (implicitly) the quantum transformation from the harmonic oscillator (even with a $1 / x^{2}$ term) to the free particle when studying the symmetries of the magnetic monopole. Junker \& Inomata (1985) [7] gave the transformation of the propagator, in a path integral approach, for a TDQH, into the free one (the equivalent of the QAT, but in terms of propagators). Takagi (1990) [8] gave the quantum transformation from the harmonic oscillator to the free particle, interpreted as the change to comoving coordinates. Bluman \& Shtelen (1996) [9] gave the (non-local) transformation of the TDSE for a TDQH plus a non-linear term into the free particle one, in the context of transformations of PDEs. Kagan et al. [10] and independently Castin \& Dum [11] (1996) introduced a scaling transformation in the Gross-Pitaevskii equation describing Bose-Einstein Condensates (BEC) which is related to the QAT. Suslov et al. (2010) [12 computed the propagator for a TDQH using the classical equations.

To the best of the authors' knowledge, the Quantum Arnold Transformation can be not only very useful to perform certain analytic calculations, but key to unify different concepts scattered through the mentioned literature in a simplified framework.

The paper is organized as follows. First, in Section 2 we revise the Classical Arnold Transformation. After that we go on with the Quantum Arnold Transformation in Section 3 . Finally two extensions are described in Section 4: the Gauged QAT and the Arnold-Ermakov-Pinney transformation.

\section{The Classical Arnold Transformation}

To set up the framework in which the classical Arnold transformation acts, let us briefly remind the subject of Lie symmetries of ordinary differential equations.

\subsection{Lie transformations}

A Lie symmetry of an ordinary differential equation (ODE) is a coordinate transformation that sends solutions into solutions. The problem of determining the Lie symmetries of an ODE is rather old, and S. Lie gave the main results at the end of the nineteenth century 2]. One of these results was that a second order differential equation (SODE) $y^{\prime \prime}=F\left(x, y, y^{\prime}\right)$ has the maximal number of Lie symmetries $(S L(3, \mathbb{R}))$ if it can be transformed to the free equation by a point transformation:

$$
y^{\prime \prime}=F\left(x, y, y^{\prime}\right) \stackrel{\substack{\tilde{x}=\tilde{x}(x, y) \\ \tilde{y}=\tilde{y}(x, y)}}{\Longrightarrow} \tilde{y}^{\prime \prime}=0 .
$$

This linearization is possible if the ODE is of the form:

$$
y^{\prime \prime}=E_{3}(x, y)\left(y^{\prime}\right)^{3}+E_{2}(x, y)\left(y^{\prime}\right)^{2}+E_{1}(x, y) y^{\prime}+E_{0}(x, y),
$$

with $E_{i}(x, y)$ satisfying some integrability conditions (see, for instance, [13, 14]).

There is a nice geometric interpretation of this condition in terms of projective geometry. The non-linear SODE (2) is obtained by projection from the geodesic equations in a 2-dim Riemannian manifold. The coefficients $E_{i}(x, y)$ are in one-to-one correspondence with Thomas projective parameters $\Pi$, and the integrability conditions that they satisfy are the conditions for the Riemann tensor to be zero (see [13, 14]). 
V.I. Arnold named this process rectification or straightening of the trajectories, and studied the case of linear SODE (LSODE), giving explicitly the point transformation for this case [1]. In the next subsection we describe it in detail.

\subsection{Classical Arnold transformation}

A General Linear Second Order Differential Equation (LSODE) is given by the differential equation:

$$
\ddot{x}+\dot{f} \dot{x}+\omega^{2} x=\Lambda,
$$

where $f, \omega$ and $\Lambda$ are functions of $t$. The Classical Arnold Transformation (CAT) is a point transformation that is a local diffeomorphism:

$$
\begin{aligned}
A: \mathbb{R} \times T & \rightarrow \mathbb{R} \times \mathcal{T} \\
(x, t) & \mapsto(\kappa, \tau)
\end{aligned}:\left\{\begin{aligned}
\tau & =\frac{u_{1}(t)}{u_{2}(t)}=\int_{t_{0}}^{t} \frac{W\left(t^{\prime}\right)}{u_{2}\left(t^{\prime}\right)^{2}} d t^{\prime} \\
\kappa & =\frac{x-u_{p}(t)}{u_{2}(t)}
\end{aligned}\right.
$$

where $T$ and $\mathcal{T}$ are, in general, open intervals containing $t_{0}$ and 0 , respectively, $u_{1}$ and $u_{2}$ are independent solutions of the homogeneous LSODE satisfying the canonicity conditions:

$$
u_{1}\left(t_{0}\right)=0=u_{2}^{\prime}\left(t_{0}\right), \quad u_{1}^{\prime}\left(t_{0}\right)=1=u_{2}\left(t_{0}\right)
$$

$u_{p}$ is a particular solution of the inhomogeneous LSODE satisfying $u_{p}\left(t_{0}\right)=u_{p}^{\prime}\left(t_{0}\right)=0$, and $W(t)=\dot{u}_{1} u_{2}-u_{1} \dot{u}_{2}=e^{-f}$ is the Wronskian of the two solutions. Here $t_{0}$ is an arbitrary time, conveniently chosen to be $t_{0}=0$ (see [3] for details).

The CAT transforms the original LSODE (3) into that of the free particle, up to a factor:

$$
\ddot{x}+\dot{f} \dot{x}+\omega^{2} x=\Lambda \quad \stackrel{A}{\longrightarrow} \quad \frac{W}{u_{2}^{3}} \ddot{\kappa}=0 .
$$

The presence of this factor implies that patches of trajectories of (3) are transformed into patches of straight (free) trajectories. In fact, an arbitrary trajectory solution of (3) can be written as $x(t)=A u_{1}(t)+B u_{2}(t)+u_{p}(t)$, and the CAT sends it to $\kappa(\tau)=A \tau+B$. While $t$ varies in the interval $T$ defined by two consecutive zeros of $u_{2}(t)$ (containing $t_{0}$ ), $\tau$ varies in the range of the map defined by $\frac{u_{1}(t)}{u_{2}(t)}$. In the case in which $u_{2}(t)$ has one zero, $T$ is (left- or right-) unbounded, and, if it has no zeros, $T$ is $\mathbb{R}$.

Even though the CAT is a local diffeomorphism, it can be defined for an arbitrary time $t_{0}$. Thus different CATs can be defined for different times $t_{0}$ and cover in this way a complete trajectory of (3). We shall show with the example of the harmonic oscillator how this can be done.

\subsubsection{The example of the harmonic oscillator}

The harmonic oscillator (HO) is the best example to understand how the CAT works. For this case, and considering $\Lambda=0$, the two solutions are $u_{1}(t)=\frac{1}{\omega} \sin (\omega t)$ and $u_{2}(t)=\cos (\omega t)$. The open interval $T$ defined by two consecutive zeros of $u_{2}(t)$, and containing $t_{0}=0$, is $\left(-\frac{\pi}{2 \omega}, \frac{\pi}{2 \omega}\right)$, and the CAT $A$ and its inverse $A^{-1}$ are then written as:

$$
\begin{aligned}
& A:\left\{\kappa=\frac{x}{u_{2}(t)}=\frac{x}{\cos (\omega t)}, \quad \tau=\frac{u_{1}(t)}{u_{2}(t)}=\frac{1}{\omega} \tan (\omega t)\right. \\
& A^{-1}:\left\{\begin{array}{c}
x=\cos (\arctan (\omega \tau)) \kappa, \quad t=\frac{1}{\omega} \arctan (\omega t) . \\
=\frac{\kappa}{\sqrt{1+\omega^{2} \tau^{2}}}
\end{array}\right.
\end{aligned}
$$


In this case $\tau \in \mathbb{R}$. Pictorially, the CAT for the HO can be represented as in Figure 1 . where velocities have also been included in the graphic for clarity. Here $A$ maps the solid part of the helix (half a period of a harmonic oscillator trajectory) into the whole line (a free particle trajectory). The horizontal plane represents the space of all possible initial conditions at $t=0=\tau$. Note that both trajectories are tangent when projected onto this plane, due to the conditions (5). See [15] for more details in this case. For the CAT to map other patches of

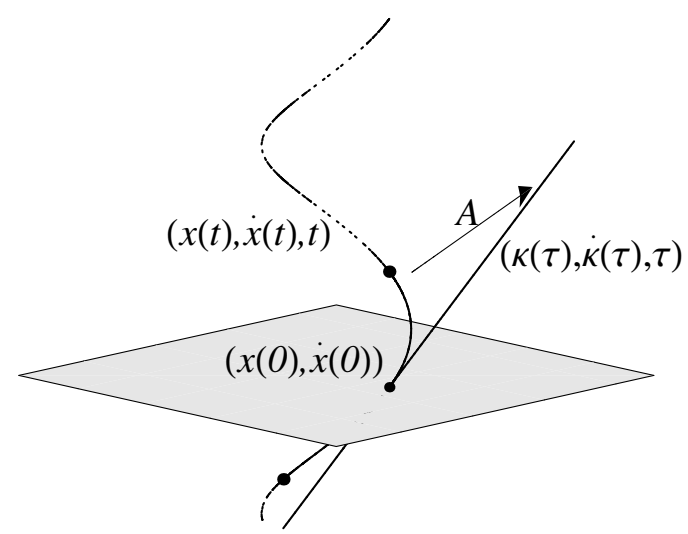

Figure 1: Depiction of the CAT for the harmonic oscillator (adapted from [15]).

the HO trajectories into the free particle trajectories, different branches of the arctan function in the inverse CAT (8) should be used (and a different $t_{0} \neq 0$ for the CAT). For each integer $k$, let us take $T_{k}=\left(\left(k-\frac{1}{2}\right) \frac{\pi}{\omega},\left(k+\frac{1}{2}\right) \frac{\pi}{\omega}\right)$ and $t_{k}=k \frac{\pi}{\omega}$. The solutions verifying conditions (5) at $t_{k}$ are $u_{i}^{(k)}(t)=(-1)^{k} u_{i}(t)=u_{i}\left(t-t_{k}\right), i=1,2$. Define a pair of CAT and inverse CAT from $\mathbb{R} \times T_{k}$ into $\mathbb{R}^{2}$ of the form: $A_{(k)}(x, t)=\left(\frac{x}{u_{2}^{(k)}(t)}, \frac{u_{1}^{(k)}(t)}{u_{2}^{(k)}(t)}\right)=(\kappa, \tau)$ and $A_{(k)}^{-1}(\kappa, \tau)=(x, t)$, where the $k$-th branch of the arctan function has been used in $A_{(k)}^{-1}$. An unfolded version of the CAT, $\tilde{A}$, can be built by joining all the patches $A_{(k)}$, defining an application that maps a complete trajectory $x(t)$ of the harmonic oscillator into a trajectory $\kappa(\tau)$ of the free particle. $\tilde{A}$ is periodic on $t$ with period $\frac{\pi}{\omega}$, although discontinuous.

Other simple examples where this construction can be done are the damped particle and the damped harmonic oscillator, see [16] for details.

\section{The Quantum Arnold Transformation}

An arbitrary LSODE system (3) can be derived from the Lagrangian (we shall take $\Lambda=0$ for simplicity but the whole formalism can be developed with $\Lambda \neq 0$, see [3]):

$$
L=\frac{1}{2} m e^{f}\left(\dot{x}^{2}-\omega^{2} x^{2}\right),
$$

and from this the Hamiltonian

$$
H=\frac{p^{2}}{2 m} e^{-f}+\frac{1}{2} m \omega^{2} x^{2} e^{f}
$$


is derived, which is known as the Generalized Caldirola-Kanai (GCK) Hamiltonian for a damped oscillator (see [3] and references therein). The case in which $\dot{f}=\gamma$ and $\omega$ are constants corresponds to the original Caldirola-Kanai Hamiltonian for a damped harmonic oscillator [17, 18], and whose corresponding Lagrangian was given for the first time by Bateman [19], after a constraint to the Bateman dual system is imposed [20]. Canonical quantization of the GCK Hamiltonian leads to the time-dependent Schrödinger equation:

$$
i \hbar \frac{\partial \phi}{\partial t}=\hat{H} \phi=-\frac{\hbar^{2}}{2 m} e^{-f} \frac{\partial^{2} \phi}{\partial x^{2}}+\frac{1}{2} m \omega^{2} x^{2} e^{f} \phi .
$$

The CAT $A$ is a local (in time) diffeomorphism between the space of solutions of the LSODE system (3) and the space of solutions of the free particle. We would like to extend it to a unitary transformation $\hat{A}$, the Quantum Arnold Transformation (QAT), between the Hilbert space of solutions $\phi(x, t)$ of the time-dependent Schrödinger equation for the GCK oscillator (11) at time $t, \mathcal{H}_{t}$, into the Hilbert space of solutions $\varphi(\kappa, \tau)$ of the time-dependent Schrödinger equation for the Galilean free particle

$$
i \hbar \frac{\partial \varphi}{\partial \tau}=-\frac{\hbar^{2}}{2 m} \frac{\partial^{2} \varphi}{\partial \kappa^{2}},
$$

at time $\tau, \mathcal{H}_{\tau}^{G}$. The desired extension is given by:

$$
\begin{aligned}
\hat{A}: \mathcal{H}_{t} & \longrightarrow \mathcal{H}_{\tau}^{G} \\
\phi(x, t) \longmapsto & \varphi(\kappa, \tau)=\hat{A}(\phi(x, t)) \\
& =A^{*}\left(\sqrt{u_{2}(t)} e^{-\frac{i}{2} \frac{m}{\hbar} \frac{1}{W(t)} \frac{u_{2}(t)}{u_{2}(t)} x^{2}} \phi(x, t)\right) .
\end{aligned}
$$

Here $A^{*}$ is the pullback of the CAT $A$, acting on functions (i.e. $A^{*}(f(x, t))=f\left(A^{-1}(\kappa, \tau)\right)$ ). The QAT can be diagrammatically represented as:

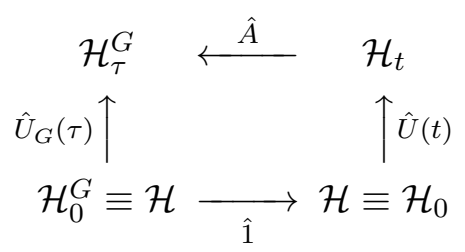

where $\mathcal{H}_{0} \equiv \mathcal{H}_{0}^{G} \equiv \mathcal{H}$ is the common Hilbert space of solutions of the Schrödinger equation for both systems at $t=\tau=0$ (we shall take, for simplicity, $t_{0}=0$, as before), $U(t)$ is the unitary time-evolution operator for the GCK oscillator and $\hat{U}_{G}(\tau)$ is the corresponding one for the Galilean free particle. The map at the bottom of the diagram is the identity due to conditions (5); otherwise a non-trivial unitary transformation appears (see [3]).

From the commutative diagram, it is clear that $\hat{A}$ is unitary given the unitarity of the evolution operators. However, it can also be checked explicitly that the scalar product of two states in $\mathcal{H}_{\tau}^{G}$ at a given time $\tau$ is the same than that of the transformed states by $\hat{A}$ in $\mathcal{H}_{t}$ at the corresponding time $t$ : 


$$
\begin{aligned}
\left\langle\varphi_{1}, \varphi_{2}\right\rangle_{\mathcal{H}_{\tau}^{G}}= & \int_{-\infty}^{+\infty} \mathrm{d} \kappa \varphi_{1}(\kappa, \tau)^{*} \varphi_{2}(\kappa, \tau) \\
= & \int_{-\infty}^{+\infty} \frac{\mathrm{d} x}{u_{2}(t)}\left(\sqrt{u_{2}(t)} e^{\frac{i}{2} \frac{m}{\hbar} \frac{1}{W(t)} \frac{\dot{u}_{2}(t)}{u_{2}(t)} x^{2}} \phi_{1}(x, t)^{*}\right) \\
& \times\left(\sqrt{u_{2}(t)} e^{-\frac{i}{2} \frac{m}{\hbar} \frac{1}{W(t)} \frac{\dot{u}_{2}(t)}{u_{2}(t)} x^{2}} \phi_{2}(x, t)\right) \\
= & \int_{-\infty}^{+\infty} \mathrm{d} x \phi_{1}(x, t)^{*} \phi_{2}(x, t)=\left\langle\phi_{1}, \phi_{2}\right\rangle_{\mathcal{H}_{t}},
\end{aligned}
$$

where $\tau, \kappa, \mathrm{d} \kappa$ and the integration limits have been transformed according to the CAT.

The QAT inherits from the CAT the local character in time, in the sense that it is valid only for $t \in T$ and $\tau \in \mathcal{T}$, although it can be defined for an arbitrary initial time $t_{0}$. To extend the QAT beyond $T$, we can proceed as in the classical case for the harmonic oscillator, considering the different branches of the inverse function of $\tau(t)$, defining an unfolded QAT, $\hat{\tilde{A}}$.

It should be stressed that if in the different branches of the unfolded CAT proper solutions verifying (5) are not used, changes in signs can appear, which result in changes in phases in the different branches of the unfolded QAT. This phenomenon is related to the Maslov correction (see for instance [21]). In fact, it can be checked that, for the case of the harmonic oscillator previously considered, $\hat{A}_{(k)}(\phi(x, t))=e^{i k \frac{\pi}{2}} \hat{A}\left(\phi\left((-1)^{k} x, t\right)\right)$.

\subsection{Symmetries of the quantum GCK oscillator}

By the results of Sec. 2.1. any classical LSODE system has the maximal number of Lie symmetries, the ones of the free particle $(S L(3, \mathbb{R}))$. The vector fields generating these symmetries can be computed by means of the CAT from the symmetries of the free particle. Even though the CAT is a local diffeomorphism, these vector fields are defined for all times [22] (the reason for this is that the only function appearing in the denominator of the vector fields is the Wronskian, which is never vanishing).

The quantum symmetries of the free Schrödinger equation 12 are smaller than $S L(3, \mathbb{R})$, only the Schrödinger group preserves 1 (12) 23]. The Schrödinger group is a semidirect product of the (centrally extended) Galilei group by the group $S L(2, \mathbb{R})$. It is generated by the basic operators:

$$
\hat{\kappa}=\kappa+\frac{i \hbar}{m} \tau \frac{\partial}{\partial \kappa}, \quad \hat{\pi}=-i \hbar \frac{\partial}{\partial \kappa},
$$

in addition to their quadratic combinations (representing scale and non-relativistic conformal transformations).

The basic operators, together with their quadratic combinations, are constant of motion operator: $2^{2}$, they are infinitesimal generators of symmetries of the free Schrödinger equation (12), and preserve the Hilbert space $\mathcal{H}_{\tau}^{G}$ of solutions 12 .

We can import the symmetries acting on the free particle Hilbert space $\mathcal{H}_{\tau}^{G}$ (the Schrödinger group) into the GCK Hilbert space $\mathcal{H}_{t}$ by means of the commutative diagram (14). The infinitesimal generators of these symmetries are:

$$
\hat{P}=-i \hbar u_{2} \frac{\partial}{\partial x}-m x \frac{\dot{u}_{2}}{W}, \quad \hat{X}=\frac{\dot{u}_{1}}{W} x+\frac{i \hbar}{m} u_{1} \frac{\partial}{\partial x},
$$

\footnotetext{
${ }^{1}$ Symmetries of the Schrödinger equation are the quantum analogue of Noether symmetries, i.e. those preserving the Lagrangian.

${ }^{2}$ They verify that $\frac{\partial \cdot}{\partial t}+\frac{i}{\hbar}\left[\hat{H}^{G}, \cdot\right]=0$, where $\hat{H}^{G}=-\frac{\hbar^{2}}{2 m} \frac{\partial^{2}}{\partial \kappa^{2}}$ is the Hamiltonian operator for the free Galilean particle.
} 
and their quadratic combinations (see [3] for the complete list of operators). All these operators expand a representation of the Schrödinger group, are constant of motion operators for (11) and preserve the Hilbert space $\mathcal{H}_{t}$.

Despite of the local character of the QAT, and similarly to the classical case, these operators are well defined for all $t \in \mathbb{R}$, since the Wronskian $W$ never vanishes.

The Hamiltonian for the GKC harmonic oscillator can be expressed in terms of the basic, conserved operators:

$$
\hat{H}=\alpha(t) \frac{\hat{P}^{2}}{2 m}+\frac{1}{2} m \beta(t) \hat{X}^{2}+\delta(t) \frac{1}{2}(\hat{X} \hat{P}+\hat{P} \hat{X}),
$$

where the time-dependent functions $\alpha(t), \beta(t)$ and $\delta(t)$ are given by:

$$
\alpha(t)=\frac{u_{1}^{2} \omega^{2}+u_{1}^{\prime 2}}{W}, \quad \beta(t)=\frac{u_{2}^{2} \omega^{2}+u_{2}^{\prime 2}}{W}, \quad \delta(t)=\frac{u_{1} u_{2} \omega^{2}+u_{1}^{\prime} u_{2}^{\prime}}{W} .
$$

Again, since the Wronskian never vanishes and the basic operators $\hat{X}$ and $\hat{P}$ are also welldefined for all times, this construction remains valid for all times.

Note that, since the basic operators $\hat{X}$ and $\hat{P}$ are conserved, $\hat{H}$ is not a conserved operator (unless $\omega=\omega_{0}$ is a constant and $\gamma=0$ ), and therefore an eigenvalue equation for it makes no sense (i.e., there is no time-independent Schrödinger equation).

Instead, any linear combination of $\hat{P}^{2}, \hat{X}^{2}$ and $\hat{X P}$ can be used to define an eigenvalue problem, and the corresponding eigenvectors would constitute a basis for the solution of the Generalized Caldirola-Kanai Schrödinger equation (see [3] and references therein).

\subsection{Applications of the QAT}

From the commutative diagram (14) and from (15) it is clear that the QAT is a unitary operator, and this has interesting and far-reaching consequences. Among them we can mention the possibility of importing operators (symmetries) from one system to the other, importing wave functions, scalar product, computing the time evolution operator, etc. We shall not discuss them here, referring the reader to [3, 15, 24] for details.

The QAT have many more and very interesting applications and generalizations. One of them is the application of the QAT to density matrices, transforming the Quantum Liouville equation of an arbitrary time-dependent quadratic Hamiltonian into the free one [15, with the possibility of extending it and to Wigner functions and other quasi-probability distributions, or even the extension of the QAT to Lindblad type equations, in order to study dissipation and decoherence under the QAT point of view. It can also be applied to Bose-Einstein Condensates, to transform the time-dependent potential (oscillator traps with time-dependent frequencies) into a time-independent potential.

The QAT can also be relevant in quantum inflationary Cosmological models, where quantum fluctuations of the scalar field are governed, under some assumptions, by a LSODE with a dissipative term.

Among the possible generalizations of the QAT, we mention the relativistic case, as the quantum version of a generalized CAT for geodesic equations in a fixed background and with external forces, or as the quantum version of geodesic mappings, that transform geodesics of a metric into geodesics of a different metric (Beltrami Theorem). Other possible generalizations refer to non-linear potentials (the Quantum Lie Transformation), to second order Riccati equations (see for instance [25]), or even to non-local potentials like in the Gross-Pitaevskii equation. 


\section{Extensions of the QAT}

In this section we shall introduce the main results of the paper: two immediate, although non-trivial, extensions of the QAT. The first one is to allow for more general Schrödinger equations than the GKC one. The second one is the transformation relating two LSODEsystems, denoted as the Arnold-Ermakov-Pinney transformation, obtained as the composition of the CAT corresponding to the first LSODE-system with the inverse of the CAT corresponding to the second LSODE-system, as well as the corresponding quantum version.

\subsection{Gauged Quantum Arnold Transformation}

The QAT, as it has been designed, transforms the solutions of the Schrödinger equation of the GKC oscillator into that of the free particle. It cannot transform, as it stands, the solutions of the Schrödinger equation associated with an arbitrary time-dependent quadratic Hamiltonian.

The reason is the choice of solutions $u_{1}(t), u_{2}(t)$ satisfying the canonicity condition (5) and the fact that the classical counterpart, the CAT, transforms the LSODE (3) into that of the free particle.

Suppose that the quantum Hamiltonian is an arbitrary, time-dependent self-adjoint quadratic Hamiltonian:

$$
\hat{H}=-\frac{\hbar^{2}}{2 m} \mu(t) \frac{\partial^{2}}{\partial x^{2}}-i \Gamma(t) \hbar\left(x \frac{\partial}{\partial x}+\frac{1}{2}\right)+\frac{1}{2} m \nu(t) x^{2},
$$

where $\Gamma(t)$ and $\nu(t)$ are real and $\mu(t)>0$ (to have a positive-definite kinetic energy).

Then the transformation that maps solutions of the Schrödinger equation for this Hamiltonian into that of the free particle is given by:

$$
\varphi(\kappa, \tau)=\hat{G}(\phi(x, t))=A^{*}\left(\sqrt{u_{2}(t)} e^{-\frac{i}{2} \frac{m}{\hbar} \frac{1}{W(t)}\left(\frac{u_{2}(t)}{u_{2}(t)}-\Gamma(t)\right) x^{2}} \phi(x, t)\right),
$$

where $u_{1}(t)$ and $u_{2}(t)$ are canonical solutions (in the sense of (5) verifying the LSODE (3) with $\dot{f}=-\frac{\dot{\mu}}{\mu}$ and $\omega^{2}=\mu \nu+\Gamma\left(\frac{\dot{\mu}}{\mu}-\Gamma\right)-\dot{\Gamma}$. The Wronskian of the two solutions is $W(t)=\mu(t)$.

Note that the transformation 21 is an ordinary QAT times an extra gauge term $e^{\frac{i}{2} \frac{m}{\hbar} \frac{\Gamma(t)}{W(t)} x^{2}}$ :

$$
\hat{G}=A^{*}\left(e^{\frac{i}{2} \frac{m}{\hbar} \frac{\Gamma(t)}{W(t)} x^{2}}\right) \hat{A} .
$$

For this reason we shall denote this transformation Gauged QAT (GQAT). In the case where $\Gamma(t)=0$, the GQAT turns into an ordinary QAT.

Note that $M(t)=\frac{m}{\mu(t)}$ can be interpreted as a time-dependent mass, and therefore the Hamiltonian can be written as

$$
\hat{H}=-\frac{\hbar^{2}}{2 M(t)} \frac{\partial^{2}}{\partial x^{2}}-i \Gamma(t) \hbar\left(x \frac{\partial}{\partial x}+\frac{1}{2}\right)+\frac{1}{2} M(t) \Omega(t)^{2} x^{2},
$$

where $\Omega(t)^{2}=\mu(t) \nu(t)$, and the classical damping coefficient and frequency are written now as $\dot{f}=\frac{\dot{M}}{M}$ and $\omega^{2}=\Omega^{2}-\Gamma\left(\frac{\dot{M}}{M}+\Gamma\right)-\dot{\Gamma}$, respectively.

\subsection{The Arnold-Ermakov-Pinney transformation}

Although the CAT relates any LSODE system with the free particle, the dynamics of both systems can be very different 3 . Thus, it could be interesting to relate directly two arbitrary

\footnotetext{
${ }^{3}$ Consider, for example, the harmonic oscillator with a bounded, periodic motion as compared with the free particle, with an unbounded non-periodic motion.
} 
LSODE systems with similar behaviour, and this can be achieved by composing a CAT and an inverse CAT.

More precisely, let $A_{1}$ and $A_{2}$ denote the CATs relating the LSODE-system 1 and LSODEsystem 2 to the free particle, respectively, then $E=A_{1}^{-1} A_{2}$ relates LSODE-system 2 to LSODEsystem 1 . $E$ can be written as:

$$
\begin{aligned}
E: \mathbb{R} \times T_{2} & \rightarrow \mathbb{R} \times T_{1} \\
\left(x_{2}, t_{2}\right) & \mapsto\left(x_{1}, t_{1}\right)=E\left(x_{2}, t_{2}\right) .
\end{aligned}
$$

The explicit form of the transformation can be easily computed by composing the two CATs, resulting in:

$$
x_{1}=\frac{x_{2}}{b\left(t_{2}\right)} \quad W_{1}\left(t_{1}\right) d t_{1}=\frac{W_{2}\left(t_{2}\right)}{b\left(t_{2}\right)^{2}} d t_{2},
$$

where $b\left(t_{2}\right)=\frac{u_{2}^{(2)}\left(t_{2}\right)}{u_{2}^{(1)}\left(t_{1}\right)}$ satisfies the non-linear SODE:

$$
\ddot{b}+\dot{f}_{2} \dot{b}+\omega_{2} b=\frac{W_{2}^{2}}{W_{1}^{2}} \frac{1}{b^{3}}\left[\omega_{1}^{2}+\dot{f}_{1} \frac{\dot{u}_{2}^{(1)}}{u_{2}^{(1)}}\left(1-b^{2} \frac{W_{1}}{W_{2}}\right)\right],
$$

and where $u_{i}^{(j)}$ refers to the $i$-th particular solution for system $j ; W_{j}, \dot{f}_{j}$ and $\omega_{j}$ stand for the Wronskian and the LSODE coefficients for system $j$; and the dot means derivation with respect to the corresponding time function.

For the particular case where LSODE-system 1 is a harmonic oscillator $\left(\omega_{1}\left(t_{1}\right) \equiv \omega_{0}\right.$ and $\dot{f}_{1}=0$ ), this expression simplifies to:

$$
\ddot{b}+\dot{f}_{2} \dot{b}+\omega_{2} b=\frac{W_{2}^{2}}{b^{3}} \omega_{0}^{2},
$$

resulting in a generalization of the Ermakov-Pinney equation. For $\dot{f}_{2}=0$ the Ermakov-Pinney equation (also known as Milne-Pinney) is recovered [26, 27, 28]:

$$
\ddot{b}+\omega_{2} b=\frac{\omega_{0}^{2}}{b^{3}},
$$

representing a harmonic oscillator of frequency $\omega_{2}$ with an extra inverse squared potential $\frac{\omega_{0}^{2}}{x^{2}}$. For $\omega_{0}=0$, the Arnold-Ermakov-Pinney transformation reduces to the ordinary CAT, i.e. $E=A$.

The Ermakov-Pinney equation is related to the Ermakov invariant [26, 4] and appears in many branches of physics, such as Cosmology [29], BEC [30], etc. Its generalization to higher dimensions, know as Ermakov pairs (or system), appears in BEC [10, 11] and what is known as Kepler-Ermakov systems [31].

The Ermakov-Pinney equation entails a kind of nonlinear superposition principle, in the sense that its solutions can be written in terms of the solutions $y_{1}(t), y_{2}(t)$ of the corresponding linear equation ( with $\omega_{0}=0$ ):

$$
b(t)^{2}=c_{1} y_{1}(t)^{2}+c_{2} y_{2}(t)^{2}+2 c_{3} y_{1}(t) y_{2}(t), \quad c_{1} c_{2}-c_{3}^{2}=\omega_{0}^{2} .
$$

The other way round, the general solution $y(t)$ of the linear equation can be writen in terms of a paricular solution $\rho(t)$ of the Ermakov-Pinney equation (28) as:

$$
y(t)=c_{1} \rho(t) \cos \left(\omega_{0} \theta(t)+c_{2}\right),
$$


where $c_{1}, c_{2}$ are arbitrary constantss and $\theta(t)=\int^{t} \rho^{-2} d t^{\prime}$. Note that this equation is just 25) for $W_{1}=W_{2}=1, t_{1}=\theta\left(t_{2}\right), \rho=b, x_{2}=y\left(t_{2}\right)$ and $x_{1}=y\left(t_{2}\right) / b\left(t_{2}\right)=c_{1} \cos \left(\omega_{0} t_{1}+c_{2}\right)$. As a result, the general solution of (28) can be determined from a particular solution $\rho(t)$ using (30) and (29).

The quantum version of the Arnold-Ermakov-Pinney transformation, $\hat{E}$, can be obtained computing the composition of a QAT and an inverse QAT, to give:

$$
\begin{aligned}
\hat{E}: \mathcal{H}_{t_{2}}^{(2)} & \longrightarrow \mathcal{H}_{t_{1}}^{(1)} \\
\phi\left(x_{2}, t_{2}\right) & \longmapsto \varphi\left(x_{1}, t_{1}\right)=\hat{E}\left(\phi\left(x_{2}, t_{2}\right)\right) \\
& =E^{*}\left(\sqrt{b\left(t_{2}\right)} e^{-\frac{i}{2} \frac{m}{\hbar} \frac{1}{W_{2}\left(t_{2}\right)} \frac{\left.\dot{b(t} t_{2}\right)}{b\left(t_{2}\right)} x_{2}^{2}} \phi\left(x_{2}, t_{2}\right)\right) .
\end{aligned}
$$

The Quantum Arnold-Ermakov-Pinney transformation is a unitary map importing solutions of a GCK Schrödinger equation from solutions of a different, auxiliary GCK Schrödinger equation which, in particular, can be the one corresponding to a harmonic oscillator. In that case the transformation is very similar to the one used in BEC, known as scaling transformation to transform the time-dependent potential (oscillator traps with time-dependent frequencies) into a time-independent harmonic oscillator potential [10, 11]. Also, in that case (i.e. for $\left.\dot{f}_{2}=0, W_{2}=1, \dot{f}_{1}=0, W_{1}=1\right)$ equation 31 reduces to the transformation given by Hartley and Ray [32] (this was already given by Lewis and Riesenfeld in [4]). However, the Quantum Arnold-Ermakov-Pinney transformation allows to choose in a suitable way the auxiliary system from which the solutions may be imported.

\section{Acknowledgments}

Work partially supported by the Fundación Séneca, Spanish MICINN and Junta de Andalucía under projects 08814/PI/08, FIS2011-29813-C02-01 and FQM219-FQM1951, respectively.

\section{References}

[1] Arnold V I, Supplementary chapters to the theory of ordinary differential equations (Nauka, Moscow, 1978); English transl., Geometrical methods in the theory of ordinary differential equations, (Springer-Verlag, New York-Berlin, 1983).

[2] Lie S (1883) Arch. Math. VIII,IX 187

[3] Aldaya V, Cossío F, Guerrero J and López-Ruiz F F (2011) J. Phys. A 44065302

[4] Lewis H R Jr. and Riesenfeld W B (1969) J. Math. Phys. 101458

[5] Dodonov V V and Man'ko V I (1979) Phys. Rev. A A20 550

[6] Jackiw R (1980) Ann. Phys. 129183

[7] Junker G and Inomata A (1985) Phys. Lett. A 110195

[8] Takagi S (1990) Prog. Theor. Phys. 841019

[9] Bluman G and Shtelen V (1996) J. Phys. A 294473

[10] Kagan Y, Surkov E L and Shlyapnikov G V (1996) Phys. Rev. A 54 R1753 
[11] Castin Y and Dum R (1996) Phys. Rev. Lett. 775315

[12] Cordero-Soto R, Suazo E and Suslov S K (2010) Ann. Phys. 3251884

[13] Mahomed F M, Qadir A (2009) J. Nonlinear Math. Phys. 16283

[14] Aminova A V and Aminov N A M (2010) Sb. Math. 201631

[15] Guerrero J, López-Ruiz F F, Aldaya V and Cossío F (2011) J. Phys. A 44445307

[16] Guerrero J, Aldaya V, López-Ruiz F F and Cossio F (2012) Int. J. Geom. Meth. Mod. Phys. 91260011 (8 pages)

[17] Caldirola P (1941) Nuovo Cimento 18393

[18] Kanai E (1948) Prog. Theor. Phys. 3440

[19] Bateman H (1931) Phys. Rev. 38815

[20] Guerrero J, López-Ruiz F F, Aldaya V and Cossío F (2012), J. Phys. A 45, 475303

[21] Horvathy P A (2011) Cent. Eur. J. Phys. 91

[22] González López A (1988) J. Math. Phys. 29 1097-5

[23] Niederer U (1972) Helv. Phys. Acta 45 802; Niederer U (1973) Helv. Phys. Acta 46 191; Niederer U (1974) Helv. Phys. Acta 47167

[24] López-Ruiz F F, Guerrero J and Aldaya V (2011) J. Russ. Laser Res. 32372

[25] Cariñena J F and de Lucas J (2011), J. Geom. Mech. 3, 1

[26] Ermakov V P (1880) Univ. Izv. Kiev 201

[27] Milne W E (1930) Phys. Rev. 3586367

[28] Pinney E (1950) Proc. A.M.S. 1681

[29] Hawkins R M and Lidsey J E (2002) Phys. Rev. D 66023523

[30] Schuch D and Kaushal R S (2011) J. Phys.: Conf. Series 306012032

[31] Leach P G L and Karasu A (Kalkanli) (2004) J. Nonl. Math. Phys. 11 269-75

[32] Hartley J G and Ray J R (1982), Phys. Rev. D 25, 382 